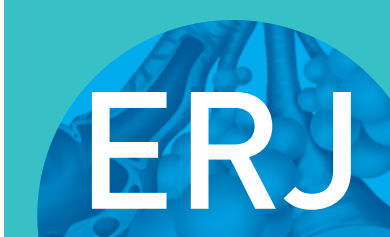

open research

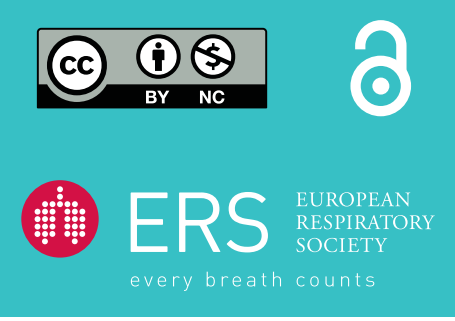

\section{Shorter time to clinical decision in work-related asthma using a digital tool}

\author{
To the Editor:
}

Occupational exposure accounts for a substantial proportion $(16 \%)$ of adult asthma $[1,2]$ and asthma may also be aggravated by triggers at the workplace. Serial measurements of lung function with a peak expiratory flow (PEF) meter may confirm work-related asthma and is recommended in US, European and Swedish national guidelines [3-7]. PEF should ideally be measured at least six times a day at the workplace and at home over a period of several weeks [8], noted down and subsequently analysed. The time-consuming and cumbersome nature of serial PEF thus limits its clinical usefulness [9]. However, this could potentially be alleviated by digital health point-of-care tools [10].

AsthmaTuner (Medituner AB, Stockholm, Sweden) is a cloud-based system for collection and analysis of lung function and symptoms using a wireless spirometer and a smartphone application [11]. We conducted a prospective nonrandomised clinical trial comparing the standard method of serial PEF to AsthmaTuner in patients with suspected asthma referred to the occupational medicine clinics at Lund, Gothenburg, Linköping, Umeå, Uppsala and Stockholm, all in Sweden.

Asthmatuner is a CE-marked system with a healthcare web interface (careportal) and a downloadable patient app (Android or iOS). It allows for portable registration of symptoms and lung function, and measurement of PEF and forced expiratory volume in $1 \mathrm{~s}$ using a Bluetooth spirometer that connects wirelessly to the patient's smartphone. Automated reminders can be set in the app. In the careportal, the data can be analysed for, for example, PEF variability and the results transferred to the electronic health record or exported for separate analysis. AsthmaTuner was developed in collaboration with Swedish patient organisations and patient focus groups, as well as the aforementioned occupational medicine clinics.

The control group consisted of patients with suspected work-related asthma referred to Occupational and Environmental Medicine, Region Skåne, in Lund, and was evaluated following their standard protocol with self-recording of data using an analogue PEF meter and 3-6-week diary. At the first meeting, the patient received information and the physician could also call the patient during the measurement period to encourage motivation. The collected data were brought physically to the clinic where the physician transferred data manually into a digital spreadsheet in which variability was calculated according to Global Initiative for Asthma (GINA) 2019 guidelines [5].

Data collection was coordinated by a study nurse, who also recorded adverse events. Lung function data were encrypted and stored on a secure cloud server (Microsoft Azure; Microsoft Corp., Redmond, WA, USA). All data handling fulfilled the requiements of the General Data Protection Regulation. The regional board of the Ethical Committee in Stockholm approved the study (identifier 2017/1536-31/2). "Clinical decision" was defined as ruling in or ruling out work-related asthma, or a decision that further examinations using other methods were needed.

The primary endpoint was time in weeks from study entry to clinical decision. The secondary endpoint was time in minutes used in the clinic to instruct the patient and analyse patient data, measured by

$@$ ERSpublications

PEF curves are a useful but cumbersome tool in diagnosing work-related asthma. Using a digital spirometer and smartphone app, time to clinical decision could be shortened by 6-7 weeks. Physician's time spent analysing PEF data is also shortened. https://bit.ly/3d5SY78

Cite this article as: Bjerg A, Ljungberg $\mathrm{H}$, Dierschke $\mathrm{K}$, et al. Shorter time to clinical decision in work-related asthma using a digital tool. ERJ Open Res 2020; 6: 00259-2020 [https://doi.org/10.1183/ 23120541.00259-2020].

Copyright $\odot$ ERS 2020. This article is open access and distributed under the terms of the Creative Commons Attribution NonCommercial Licence 4.0. 
stopwatch. Data are presented as mean \pm SE and two-sided p-values for comparison of means are shown. Data were analysed using Microsoft Excel (Microsoft Corp.) and Medcalc (medcalc.org).

The time requirements for the standard serial PEF procedure carried out in Lund $(n=10)$ was compared with an AsthmaTuner sample in Lund $(n=10)$ and to all AsthmaTuner participants $(n=30)$, which included the Lund AsthmaTuner sample. Time to clinical decision was significantly shorter in the AsthmaTuner samples: mean $4.1 \pm 0.73$ weeks in the Lund sample and $5.7 \pm 0.73$ weeks in all participants compared with standard serial PEF, mean $12.4 \pm 2.9$ weeks; $\mathrm{p}=0.012$ and $\mathrm{p}=0.002$, respectively (figure 1a).

In addition, physicians spent less time when using AsthmaTuner compared with standard serial PEF: mean $57 \pm 8.9 \mathrm{~min}$ in the Lund sample and $77 \pm 7.0 \mathrm{~min}$ in all AsthmaTuner participants versus $103 \pm 6.0 \mathrm{~min}$ using the standard method; $\mathrm{p}<0.001$ and $\mathrm{p}=0.045$, respectively (figure $1 \mathrm{~b}$ ). This was due to significantly less time needed for data analysis using AsthmaTuner: $56 \mathrm{~min}(\mathrm{p}<0.001)$ less in Lund and $30 \mathrm{~min}$ $(p=0.024)$ less in the full AsthmaTuner sample. The time spent on instructing the patient was slightly longer ( 9 min) using AsthmaTuner $(\mathrm{p}=0.025)$ in the Lund sample but not different when measured in all AsthmaTuner participants $(\mathrm{p}=0.195)$.

Our results showed that the digital instrument AsthmaTuner was able to shorten the time until clinical decision by 7-8 weeks compared with standard serial PEF curves, while also demanding less of the physician's time. This has several potential advantages. For the patient, longer time to clinical decision may delay treatment, and increase the risk of sick leave, and for continued and unnecessary harmful exposure [12]. Also, remembering to repeatedly recording data and keeping track of the equipment and PEF logs over prolonged time periods is both cumbersome and error prone. In addition, reporting bias is less likely using AsthmaTuner. With standard serial PEF, the patients have to fill their recorded flow rates in a table, and as it is easy to forget to blow, some patients may fill in an assumed value.

For the clinician and healthcare system, time is costly and poor compliance/loss-to-follow-up as well as errors in data recording, transfer and manual analysis all introduce diagnostic uncertainty. Most of these drawbacks could be mitigated by using a cloud-based digital tool like AsthmaTuner. Its advantages include significantly shorter time in the clinic, automatic reminders, and automatic storage, transfer, analysis and presentation of data. In addition, results can also be exported for documentation purposes or research.

Limitations of this study include lack of randomisation and the inherently nonblinded nature of the intervention. The control group was rather small and from only one centre (Lund). It is known that routines for, for example, how often PEF should be measured and the length of the measurement period differ between caregivers. For this reason, we made separate comparisons with AsthmaTuner patients in Lund, as well as with the whole nationwide sample. The lack of randomisation may introduce recruitment bias; however, it seems unlikely that the patients requiring the longest investigations and most time for data analysis should systematically have declined the AsthmaTuner option. While most clinicians were able to start using AsthmaTuner without prior training, physicians being new to the system may have caused overestimation of the time required. This, if anything, would have caused an underestimation of the observed differences, increasing the risk of a Type II error. No adverse events were recorded.

Serial PEF measurement is an important diagnostic tool in work-related asthma [3, 13]. Our study does not allow any conclusions to be drawn regarding whether AsthmaTuner affected diagnostic accuracy. Since
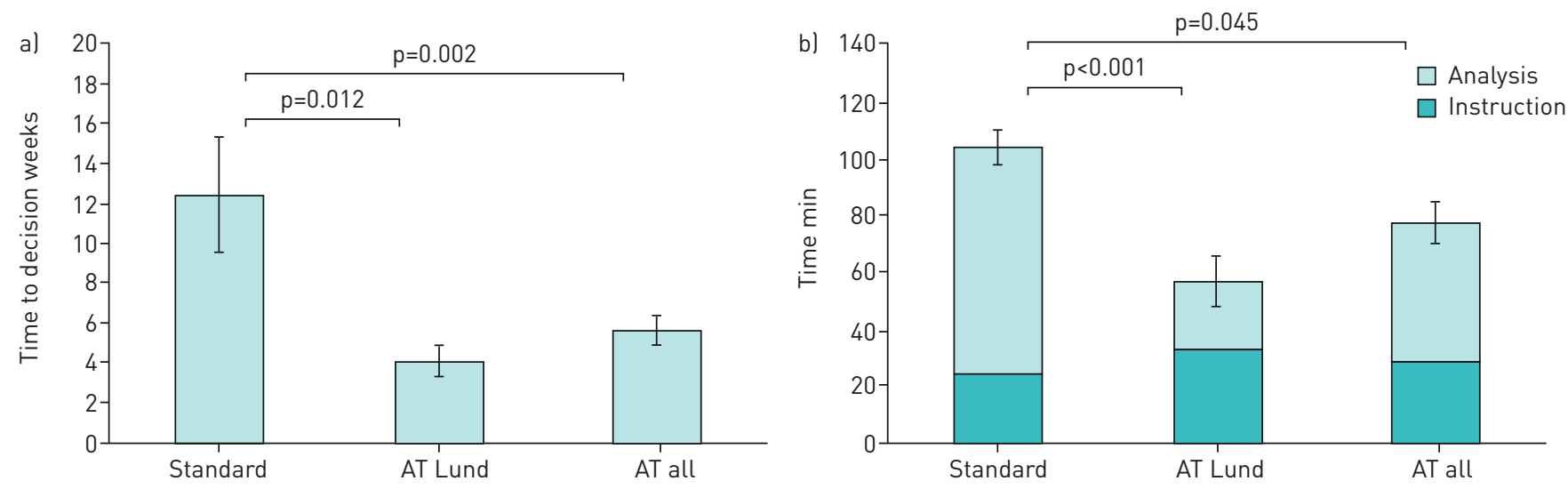

FIGURE 1 Time requirements for standard serial PEF versus AsthmaTuner (AT) in Lund and the nationwide sample. a) Time in weeks from study entry to final clinical decision and b) time in minutes spent in the clinic instructing the patient and analysing patient data. Standard error bars and p-values for test of difference of means. 
the same GINA 2019 guidelines were used in both intervention and control groups, we expect this to be similar. One previous trial concluded that AsthmaTuner improved asthma control (Asthma Control Test score) among adult patients in primary care and paediatric patients with uncontrolled asthma [11]. Moreover, a Dutch study recently indicated that cost-effectiveness of traditional asthma care can be improved by partly replacing monitoring with eHealth solutions [14].

We conclude that compared with standard methods, AsthmaTuner was significantly more time-effective in obtaining and analysing serial PEF in work-related asthma. It could be expected that using this method, serial PEF would become more attractive as a diagnostic tool in other asthma as well.

Anders Bjerg $\oplus^{1,2}$, Henrik Ljungberg ${ }^{1,3,4}$, Katrin Dierschke $\odot^{5}$, Else Åkerberg-Krook ${ }^{5}$, Ulla B.K. Andersson ${ }^{5}$, Anna-Carin Olin $\odot^{6}$, Elisabeth Lindberg ${ }^{7}$, Ximing Yuan $\odot^{7}$, Jonathan Lyström ${ }^{8}$, Robert Wålinder $\odot^{9}$, Susanne Victor ${ }^{9}$, Martin Andersson ${ }^{10}$, Helén Bertilsson ${ }^{10}$, Anna Carleborg ${ }^{1,4}$ and Björn Nordlund $\odot^{1,3,4}$

${ }^{1}$ Dept of Women's and Children's Health, Karolinska Institutet, Stockholm, Sweden. ${ }^{2}$ Martina Children's Hospital, Stockholm, Sweden. ${ }^{3}$ Lung-Allergy Dept at Astrid Lindgren Children's Hospital, Karolinska University Hospital, Stockholm, Sweden. ${ }^{4}$ MediTtuner AB, Stockholm, Sweden. ${ }^{5}$ Occupational and Environmental Medicine, Region Skåne, Kristianstad, Sweden. ${ }^{6}$ Occupational and Environmental Medicine, University of Gothenburg, Gothenburg, Sweden. ${ }^{7}$ Occupational and Environmental Medicine Centre, Dept of Health, Medicine and Caring Sciences, Linköping University, Linköping, Sweden. ${ }^{8}$ Centre for Occupational and Environmental Medicine in Stockholm, Region Stockholm, Stockholm, Sweden. ${ }^{9}$ Occupational and Environmental Medicine, Uppsala University, Uppsala, Sweden. ${ }^{10}$ Dept of Public Health and Clinical Medicine, Sustainable Health, Umeå University, Umea, Sweden.

Correspondence: Anders Bjerg, Martina Children’s Hospital, Valhallav. 91F, SE-11486, Stockholm, Sweden. E-mail: anders.bjerg@bsmartina.se

Received: 7 May 2020 | Accepted: 18 May 2020

Acknowledgements: We acknowledge all the study participants and all the clinical staff involved in this study at occupational medicine clinics in Lund, Gothenburg, Linköping, Umeå, Uppsala and Stockholm.

Data availability: All collected individual data that underlie the results reported in this article are available and will be shared after deidentification. The study protocol and statistical analysis plan, informed consent, clinical study report, and data set with analytical code will be available immediately after publication. Data will be available for investigators whose proposed use of the data has been approved by an independent review committee identified for this purpose and for any type of analyses. Proposals may be submitted up to 36 months following article publication. To gain data access, data requestors should be directed to B. Nordlund (bjorn.nordlund@ki.se) and will need to sign data access agreement. All data are stored for 10 years at Karolinska Institutet, Sweden.

Conflict of interest: A. Bjerg has nothing to disclose. H. Ljungberg is a cofounder of and holds shares in the company Medituner, which owns Asthmatuner. K. Dierschke has nothing to disclose. E. Åkerberg-Krook has nothing to disclose. U.B.K. Andersson has nothing to disclose. A-C. Olin reports that she is a founder, chair holder and board member of PExA AB outside the submitted work. In addition, Dr. Olin has patents WO2009045163 (Collection and measurement of exhaled particles) issued and WO2013117747 (A device and method for non-invasive analysis of particles during medical ventilation) pending. E. Lindberg has nothing to disclose. X. Yuan had nothing to disclose. J. Lyström has nothing to disclose. R. Wålinder has nothing to disclose. S. Victor has nothing to disclose. M. Andersson has nothing to disclose. $\mathrm{H}$. Bertilsson has nothing to disclose. A. Carleborg receives a salary from Medituner AB as project leader. B. Nordlund is a cofounder of and holds shares in the company Medituner, which owns Asthmatuner.

Support statement: The study was supported by the Sweden's Innovation Agency (Vinnova) Medtech4Health, Stockholm County Council ALF-Medicine and Medituner AB.

\section{References}

1 Kogevinas M, Zock JP, Jarvis D, et al. Exposure to substances in the workplace and new-onset asthma: an International Prospective Population-based Study (ECRHS-II). Lancet 2007; 370: 336-341.

2 Blanc PD, Annesi-Maesano I, Balmes JR, et al. The occupational burden of nonmalignant respiratory diseases. An Official American Thoracic Society and European Respiratory Society Statement. Am J Respir Crit Care Med 2019; 199: 1312-1334.

3 Trivedi V, Apala DR, Iyer VN. Occupational asthma: diagnostic challenges and management dilemmas. Curr Opin Pulm Med 2017; 23: 177-183.

4 Moscato G, Godnic-Cvar J, Maestrelli P, et al. Statement on self-monitoring of peak expiratory flows in the investigation of occupational asthma. Subcommittee on Occupational Allergy of the European Academy of Allergology and Clinical Immunology. American Academy of Allergy and Clinical Immunology. European Respiratory Society. American College of Allergy, Asthma and Immunology. Eur Respir J 1995; 8: 1605-1610.

5 The Global Initiative for Asthma. Global Strategy for Asthma Management and Prevention. GINA, 2019.

6 Guidelines for Health Care of Asthma and COPD. Stockholm, The National Board of Health and Welfare in Sweden, 2017. 
7 U.S. Department of Health and Human Services, National Heart, Lung, and Blood Institute. Expert Panel Report 3 (EPR-3): Guidelines for the Diagnosis and Management of Asthma; Summary Report 2007. J Allergy Clin Immunol 2007; 120: S94-S138.

8 Anees W, Gannon PF, Huggins V, et al. Effect of peak expiratory flow data quantity on diagnostic sensitivity and specificity in occupational asthma. Eur Respir J 2004; 23: 730-734.

9 Reddel H, Jenkins C, Woolcock A. Diurnal variability - time to change asthma guidelines? BMJ 1999; 319: 45-47.

10 Slader CA, Belousova EG, Reddel HK. Measuring peak flow enhances adherence to monitoring in asthma. Thorax 2007; 62: 741-742.

11 Ljungberg $\mathrm{H}$, Carleborg A, Gerber $\mathrm{H}$, et al. Clinical effect on uncontrolled asthma using a novel digital automated self-management solution: a physician-blinded randomised controlled crossover trial. Eur Respir J 2019; 54: 1900983.

12 Miedinger D, Malo JL, Ghezzo H, et al. Factors influencing duration of exposure with symptoms and costs of occupational asthma. Eur Respir J 2010; 36: 728-734.

13 Pralong JA, Cartier A. Review of diagnostic challenges in occupational asthma. Curr Allergy Asthma R 2017; 17: 1.

14 van den Wijngaart LS, Kievit W, Roukema J, et al. Online asthma management for children is cost-effective. Eur Respir J 2017; 50: 1701413. 